\title{
MASCULINIDAD Y TRABAJO INDUSTRIAL. UNA EXPLORACIÓN DE SUS VÍNCULOS
}

\author{
Masculinity and industrial work. An exploration of their links
}

Carla Aguilar-Cunill* Ignasi Brunet ICART

Universidad Rovira i Virgili

\begin{abstract}
RESUMEN
Este artículo forma parte del marco teórico de una investigación sobre las construcciones de género en la industria química. El objetivo es analizar los fuertes vínculos históricos de la masculinidad con el trabajo industrial, que se funden en una misma lógica productiva, así como en las competencias y capacidades que deben tener las personas trabajadoras. También plantea cómo esta relación se aplica sobre las mujeres, siendo la feminidad un elemento tradicionalmente excluido del espacio de trabajo industrial. Pese a que el análisis está centrado en la variable género, integramos también una perspectiva interseccional, teniendo en cuenta variables como la clase social o la orientación sexual.

Palabras clave: género, trabajo, masculinidad, feminidad, industria.
\end{abstract}

\section{ABSTRACT}

This article is part of the theoretical framework in an empirical research about gender constructions in the chemical industry. The aim is to analize the historical strong links between masculinity and industrial work, wich are melt in the same productive logic, as well as in the competences and capacities that workers need to have. Also propose how this relationship applies to women, being feminity an element tradicionally excluded in the field of the industrial work. Although the analysis is centered in the gender variable, it incorporates an intersectional perspective too, considering variables as social class or sexual orientation.

Keywords: Gender, work, masculinity, feminity, industry.

* Correspondencia a: Carla Aguilar-Cunill. Av. Catalunya, 35. 43002 Tarragona - carla.aguilar@urv.cat - http://orcid.org/00000003-4148-2264

Cómo citar: Aguilar Cunill, Carla; Brunet Icart, Ignasi. (2018). «Masculinidad y trabajo industrial. Una exploración de sus vínculos"; Lan Harremanak, 40, 115-137. (https://doi.org/10.1387/lan-harremanak.20393).

Recibido: 14 noviembre, 2018; aceptado: 3 diciembre, 2018.

ISSN 1575-7048 - elSSN 2444-5819 / (c) 2018 UPV/EHU

(c) (i) Esta obra está bajo una licencia

Creative Commons Atribución 4.0 Internacional 


\section{SUMARIO}

1. Introducción.-2. Metodología.-3. Género y mercado de trabajo.—4. La masculinidad (obrera) en el entorno laboral industrial. 4.1. La racionalización masculina del trabajo. 4.2. La construcción del obrero en masculino. 4.3. La masculinidad como competencia laboral. 4.4. Riesgo y violencia en el entorno industrial.-5. Las dificultades de incorporar a las mujeres en el ámbito del trabajo industrial. 5.1. La exclusión de la feminidad. 5.2. La masculinización como resolución de los conflictos entre género e identidad laboral en las mujeres.-6. Conclusiones.-7. Referencias bibliográficas.

\section{Introducción}

En el patriarcado, la estructura social está codificada por la división sexual del trabajo. Una división que también es un sistema de poder, ya que establece las jerarquías de estatus y poder (Beard, 2018), y legitimado mediante la diferencia sexual, que establece una vinculación estable entre cuerpo y género, asumiendo que ambos son estables, de manera que el hombre se identifica por la masculinidad y la mujer, por la feminidad (Butler, 2007). Además, mediante la diferencia sexual se disfrazan de naturales y se revisten de inevitabilidad y necesidad los géneros y sus cualidades, trampa esencialista de adoctrinamiento masivo mediante la cual se moldean hombres y mujeres, y se inculca una normatividad de género, para la cual únicamente hay dos géneros, y en la que lo «natural» es la heterosexualidad y que el sexo «normal» es el sexo reproductivo (Morini, 2014). Es un modelo binario que forma parte de la operación ideológica de «recubrir de naturaleza lo que es un constructo social y cultural atravesado por el poder y perpetuador del poder» (Serra, 2018: 47), y que confirma, según Serra, la tesis de Foucault $(1999,2007)$ y de Laclau (2000) de que el individuo no puede anteceder al poder, imposibilitando de hecho la existencia de un antes del poder. Las mujeres y los hombres son sujetos producidos por un sistema de poder: el sistema de género. Sistema de control patriarcal que mediante la diferencia sexual organiza las identidades y los roles de género, al margen de que cumplan o no los modelos de género, de masculinidad y feminidad normativa, socialmente previstos para los hombres y las mujeres.

En el capitalismo industrial, el sistema de género contribuyó fuertemente a reforzar la práctica de la heterosexualidad y la heteronormatividad, siendo en muchos contextos sociales e históricos obligatoria, de acuerdo al marco le- 
gal, y sostenida a nivel simbólico por la creencia de la complementariedad entre hombres y mujeres. Una práctica de la que son objeto los hombres y las mujeres, y que decide con qué atributos genéricos dotar a "lo masculino» y a "lo femenino", y ello en un sistema binario de contrarios. Mediante esta práctica adquiere sentido la división sexual del trabajo, mediante la que se asigna el trabajo llamado reproductivo al género femenino, y el trabajo llamado productivo al grupo social dominante (al género masculino), «que en realidad es el trabajo asalariado no relacionado con el hogar y con el cuidado" (Pazos, 2018: 44). Un trabajo que crea la familia como una formación social jerárquica, donde el hombre es el patrón y la mujer realiza todo el trabajo de reproducción en el orden heteropatriarcal (Esteban, 2011; Federici, 2013; Claveria, 2018).

Un trabajo que permite hablar a Federici (2010) de un patriarcado del salario, de un sistema de poder al que sólo se accede desde la heterosexualidad, y que con un solo salario moviliza al hombre y también a la mujer que reproduce la fuerza de trabajo. De manera que, mediante la jerarquización que permite el género, el trabajo reproductivo, invisible y no remunerado de las mujeres resulta incluido en el trabajo productivo de los hombres. Federici, en Tzul (2015) resalta que en este patriarcado, cobra uno, que es también quien recibe el reconocimiento social, pero se realizan dos trabajos. Es en este sentido en el que esta autora defiende que el matrimonio es un sistema también laboral, ya que el salario permite sostener a la familia y crear el espacio en el que las mujeres producen y reproducen a los trabajadores. $\mathrm{Y}$ es que desde la investigación feminista se cuestiona la existencia de una identidad de género nuclear, cuyos significados estructuren la estructura socio-laboral de la sociedad. De forma que el objetivo final es analizar la forma en la que se da la ilusión de que el género está materialmente fundamentado (Badinter, 1993; Lamas, 1994, 2008; Butler, 2006; SoleyBeltran, 2009; Preciado, 2016; Blanco-Cano, 2017).

Ante esto, es fundamental entender los mecanismos mediante los cuales se construye la diferencia sexual y, concretamente, los mecanismos con que se produce la materialidad del sexo, una norma, según Butler (2002), que materializa cuerpos con estándares normativos de identidad, y que funcionan conectados con la división sexual del trabajo. División que opera como un lenguaje en la medida en que se emplea como un conjunto «de prácticas atributivas, explicativas y predictivas» (Butler, 2006). Es esta división la que ha comportado dificultades y conflictos a la incorporación de la mujer al mercado de trabajo remunerado. Procesos que han sido diferentes para cada sector laboral, y que responden a relaciones de poder que dinamizan «nociones que configuran posiciones de género en los trabajadores" (Palermo, 2018: 106), y que explican que actualmente el ámbito del trabajo industrial esté mayormente ocupado por hombres, y donde la brecha de género es mayor, según datos del Banco Mundial para el año 2017. Siguen siendo entornos laborales en los que los trabajadores se comprometen en 
una posición de género (Connell, 1995; Kimmel, 1997; Fuller,1997; Gilmore, 1994; Mosse, 2001; Nash, 2014; Collins, 2015), que requiere de habilidades y competencias tradicionalmente atribuidas a lo que la sociedad occidental define como masculinidad hegemónica, como la fuerza o la asunción de riesgos (Sanchez-Palencia e Hidalgo, 2001; Segarra y Carabí, 2000; Seidler, 2006; Palermo, 2015, 2017; Salinas, 2016). Masculinidad bajo la cual subyacen otras que son subalternas y que el futuro "de la masculinidad es una plétora de masculinidades» (Perry, 2018: 149), justamente para visibilizar y desmontar el monopolio masculino, blanco, burgués y heterosexual (Serra, 2018).

En tanto que el patriarcado es un sistema de poder y en el que las relaciones de poder se estructuran alrededor de determinadas concepciones de género (Comas, 1995, 1995a; Archetti, 1999; List Reyes, 2005), hemos efectuado el presente estado de la cuestión, vinculado a una investigación empírica en curso, con el objetivo de conocer la forma o formas en que el proceso laboral industrial se estructura también alrededor de determinadas concepciones de género. Concepciones que imponen un determinado tipo de masculinidad, que «lejos de ser algo con lo que se nace, se aprende en los espacios en los que transitamos» (Palermo, 2018: 113). Un aprendizaje que se diseña «en las sociedades occidentales/capitalistas sobre su negativo o lo abyecto: lo femenino», que, como norma, actúa «como una frontera denigrante de lo masculino, un recordatorio constante de aquello que nos genera rechazo» (Palermo,2018: 113-114). Una frontera que ha contribuido, por un lado, a la reafirmación de la masculinidad a través del deseo heterosexual y, por otro, ha influido en la poca presencia de mujeres, al definir como sujetos viables, no abyectos, las categorías centrales de hombre y heterosexual, que se mantienen por la continua performatividad o performance, y que excluyen la categoría mujer. Esta práctica social se asienta sobre la «naturalización» de la diferencia sexual (Butler, 2002; Huerta, 1999; Gil Calvo, 2006; Zerilli, 2008; Valdés y Olavarría, 1997,1998; Embrick et al., 2007; Claveria, 2018; Brunet y Santamaria, 2018).

\section{Metodología}

Nos apoyamos en una metodología basada en el análisis e interpretación de textos tanto teóricos como empíricos, y desde una perspectiva histórica, apoyándonos también en la perspectiva de la interseccionalidad. Todo ello para enmarcar un estudio de caso sobre el género en la industria química, por lo que nos basaremos en investigaciones contextualizadas en el mundo occidental, especialmente en Europa y América, entendiendo que las culturas del trabajo vinculadas al género en estos países, pese a sus diferencias territoriales, tienen unas raíces comunes que, en su desarrollo, siguen compartiendo. 
La perspectiva interseccional, de acuerdo con Kelan (2009: 185), se refiere a que la investigación en interseccionalidad ha mostrado "que es difícil de distinguir qué identidad es más importante en una situación», y, más bien, ha de remarcar «el hecho de que la interseccionalidad crea formas específicas de opresión». De hecho, ya que, de acuerdo con Crenshaw (1991: 1242), la interseccionalidad «realza la necesidad de tener en cuenta múltiples fundamentos de la identidad cuando consideramos como está contruido el mundo social». Ello es especialmente evidente al analizar las construcciones de género en el ámbito del trabajo industrial, muy vinculadas a la variable clase social y sus construcciones identitarias. De ahí que haya sido imprescindible tener en cuenta elementos como la identidad obrera o las formas de explotación industrial de la fuerza de trabajo para profundizar en los modelos de género. Pese a ello, la dificultad metodológica que aparece al aplicar la perspectiva interseccional en los trabajos empíricos, unido a la amplia bibliografía específica de género en el entorno laboral, ha hecho que buena parte de los trabajos aquí utilizados hayan sido análisis centrados únicamente en una de las variables analizadas. Por otro lado, la perspectiva histórica nos ha permitido observar la evolución de las relaciones entre la industria y el género, haciendo especial hincapié en sus raíces tras la revolución industrial, pero también observando sus efectos en la actualidad.

\section{Género y mercado de trabajo}

El ámbito laboral no es neutro al género, sino todo lo contrario: en la misma base de su estructura yace el sistema de género. Sistema patriarcal que determina que las dinámicas del mercado de trabajo estén profundamente generizadas (Acker, 2000; Pérez-Orozco, 2014). Buen ejemplo de ello, es la consideración del trabajo femenino como menos importante, y que aparece en diversas investigaciones como causa subyacente de la discriminación laboral femenina por parte de las empresas (Stobbe, 2005; Peterson, 2007; Longo, 2009). Ello muestra que, pese a los cambios en el mercado de trabajo que lo hacen cada vez más flexible, precarizado y disperso (Romero, 2003), se mantenga una organización patriarcal que reproduce la dominación masculina, otorgando a los hombres privilegios horizontales y verticales, a la vez que manteniendo los conflictos de identidad que aparecen cuando se trunca el modelo breadwinner (PinedaDuque, 2000).

Otro ejemplo, es el hecho de que el trabajo mismo se constituya de forma diferente cuando se considera masculino o femenino (Maruani, 2000). Siguen existiendo trabajos considerados como propios de mujeres y hombres, que se construyen simbólicamente de forma antagónica. Analizando distintas investigaciones, observamos cómo el trabajo de los hombres es construido como trabajo "duro" (Matošević, 2010) mientras el de las mujeres es visto como trabajo 
«blando» (Peterson, 2007), atributos que se trasladan a sus actores: hombres duros y mujeres suaves. La referencia a la dureza en los trabajos masculinos se usa en sus diferentes acepciones, desde la dureza física a la que suponen los horarios o el cansancio mental. De hecho, se mantiene la consideración de trabajo duro en las profesiones en las que desaparece el uso de la fuerza, unido a su mayor consideración social. El hecho de que un trabajo sea considerado masculino o femenino está fuertemente conectado con su nivel de estatus y salario (Wikander, 2016), hasta el punto de ser un indicador de proletarización o devaluación social (Téllez, 2001). En este punto, debemos señalar la problematización que desarrolla Collins (2015) en torno a la distinción entre trabajos femeninos y masculinos, en el sentido que simplifican la realidad y reproducen una visión dicotómica del género. Como bien desarrolla el autor, si analizamos en profundidad los entornos de trabajo masculinizados, vemos que en ellos no se privilegia a los hombres en general, sino a un conjunto determinado de hombres que siguen las normas de la masculinidad imperantes en ese entorno laboral.

Desde la perspectiva de las personas trabajadoras, vemos cómo el género afecta profundamente a sus trayectorias laborales, que presentan importantes divergencias entre hombres y mujeres (Longo, 2009). Ello se puede observar especialmente en los momentos de contratación y de despido, en los que la masculinidad aparece como un elemento casi necesario para que un trabajador sea competente en muchos casos. Incluso los hombres que trabajan en puestos tradicionalmente feminizados reconocen que su género ha sido un elemento discriminante en términos positivos (Simpson, 2004). Y es que la naturalización de unas determinadas capacidades y habilidades en hombres y mujeres subyace en las decisiones de las empresas sobre las personas trabajadoras. En los estudios de Stobbe (2005) y Peterson (2007), vemos cómo la masculinidad de los trabajadores es un elemento determinante en su contratación y en su despido, respectivamente. En ambos casos, se identifican unas construcciones de la masculinidad que coinciden con las características consideradas necesarias en el lugar de trabajo. Lo que, a su vez, genera el discurso inverso, observado en ambas investigaciones: que ese trabajo en concreto no es adecuado para las mujeres.

\section{La masculinidad (obrera) en el entorno laboral industrial}

\subsection{La racionalización masculina del trabajo}

A través de la racionalización del proceso productivo y el desarrollo de las competencias, como elemento clave en la definición de un puesto de trabajo, las características propias de la masculinidad se construyen como centrales en las concepciones de la persona trabajadora, especialmente en los entornos laborales tradicionalmente masculinos, como el sector industrial. De hecho, la revolución 
industrial aportó una importante novedad: la organización colectiva de la producción, lo que comportó la concentración de grandes números de trabajadores. Esta concentración se reorganizó entre finales del siglo XIX y principios del $\mathrm{xx}$, mediante el taylorismo-fordismo, que comportaba unos procesos de racionalización/burocratización de los procesos de producción. Dicha racionalización estaba asentada en la reorganización científica y la burocratización weberiana, fragmentando las tareas de producción anteriormente artesanales hasta niveles fácilmente realizables por cualquier trabajador. Este proceso implicó también que los seres humanos debían «ser motivados, manejados y escogidos para adecuarse al trabajo» (Acker, 2000: 128), para adecuarse a un puesto de trabajo y a unas tareas.

Esta racionalización descansa en el dualismo cartesiano cuerpo-mente, dualismo sobre el que se fundamenta buena parte de la lógica de la sociedad moderna occidental, y que estructura la división hogar y fábrica, emoción y razón instrumental, se encuentra la base para idear un sujeto adecuado para el trabajo industrial y otro no adecuado para este. Esta dicotomía se estructura en torno al género, concibiendo a los hombres como los más adecuados para el trabajo industrial y, por lo tanto, regulando y normalizando su presencia y problematizando la de las mujeres. De manera que se «naturalizan» distintas capacidades de éxito en el ámbito productivo en función de los sexos, lo cual supone la «construcción de la justificación ideológica de la desigualdad» (Téllez, 2001: 8).

El proceso del cambio de la organización del trabajo artesano al industrial es descrito por Guash (2006) como un proceso de colonización, en el que el taylorismo se apodera de los saberes artesanos, transfiriéndolos a la dirección, y que organiza el proceso de trabajo de tal forma que los trabajadores sean meros ejecutores de órdenes (Coriat, 1982, 1992). De esta manera, mientras los directivos tienen situaciones laborales más estables, se imponen unas condiciones de trabajo más inestables, duras y peligrosas a los trabajadores (Matošević, 2010), a los que se atribuía un estado salvaje, alejado de la racionalidad burguesa. En este caso, la división cuerpo-mente aparece trasladado al cuerpo social, identificando a la clase burguesa con la mente y a la clase trabajadora con el cuerpo (Federici, 2010). En este marco se observa cómo la racionalización del proceso productivo en el ámbito industrial se da de forma muy generizada, integrando las habilidades y capacidades propias del entorno laboral con un modelo de masculinidad normativa propio de ese entorno laboral. Así, «mientras se estaban definiendo las organizaciones como máquinas sexualmente neutras, los principios masculinos dominaban sus estructuras de autoridad» (Kanter, 1977: 46)

\subsection{La construcción del obrero en masculino}

La construcción social del sujeto obrero tiene lugar, históricamente, en el ámbito del trabajo industrial. Fue en las fábricas donde se hizo posible esa iden- 
tidad colectiva que dio paso al obrerismo y a las acciones colectivas y organizaciones sindicales en las que se ha fundamentado la historia del movimiento obrero (Moral y Brunet, 2018). Esta identidad obrera nace en oposición a las identidades de los gremios de artesanos pre-industriales y en oposición a la identidad (y masculinidad) burguesa (Arbaiza, 2014). Y esta construcción del sujeto obrero o trabajador también es indesligable de la masculinidad, ya que «los cuerpos de hombres, la sexualidad y las relaciones con la procreación y el trabajo asalariado se funden en la imagen del obrero" (Acker, 2000: 111). Actualmente, el trabajo industrial sigue siendo una fuente de identidad de clase (Slutskaya et al, 2016: 168), ya que reúne las condiciones de fuerte división del trabajo entre manual y no manual, su vinculación a unos roles propios del modelo breadwinner y una practicidad en la comprensión del trabajo y de las tareas que implica.

Las habilidades requeridas en el entorno del trabajo industrial son reivindicadas como elemento de poder por los trabajadores, que identifican estas habilidades laborales como muestras de masculinidad. De aquí que la masculinidad de la clase obrera hizo suya la bandera de la fuerza y del dominio de la naturaleza como señas distintivas (Matošević, 2010) y en los entornos laborales industriales habitualmente encontramos «actos de carácter físico y de camaradería masculina mediante deportes de contacto, "cotilleo», relatos de contenido sexual, la habililidad de beber mucho alcohol, la expresión de deseo heterosexual y de lenguaje homofóbico» (Ward, 2015: 2017).

Esta masculinidad obrera estaría vinculada también en la actualidad con los elementos del «trabajo duro, la honestidad, la autodisciplina y capacidades de resistencia física» (Slutskaya et al, 2016: 167). Elementos que son muy positivos para el beneficio empresarial, ya que permiten el aumento de la producción a partir de una presión mediada por la muestra de masculinidad, no por factores coercitivos externos. Así, siguiendo a Connell (1995: 9), podemos afirmar que la actual economía capitalista «que trabaja mediante una división por género del trabajo es, necesariamente, un proceso de acumulación de género" y no exclusivamente un proceso de acumulación de capital económico debido a los puestos de privilegio y poder que mantienen los hombres para su género. Pero también podemos afirmar que el proceso de acumulación capitalista se vale de la masculinidad como herramienta para maximizar las plusvalías. Argumentación que explica Palermo (2015: 109) y que lleva al extremo de que los trabajadores «terminan siendo prisioneros de una disciplina fabril que se vale de la masculinidad para garantizar un trabajador resistente, resolutivo e invulnerable».

Ness (2012: 667), también, defiende esta idea, considerando que las direcciones de las empresas no son ajenas a esta dinámica, sino que «sin duda, aún sigue siendo útil para las empresas de construcción que tácitamente animan la exagerada cultura local del macho dominante». Así, a través de la masculinidad, las empresas capitalistas no solamente aumentan la producción, sino que tam- 
bién se aseguran que los intereses de los trabajadores, centrados en defender su masculinidad, serán los mismos que los de la empresa en su voluntad de aumentar la productividad, evitando u ocultando así el antagonismo por clase social. Un ejemplo muy gráfico de esta estrategia es el que describe Matošević (2010) a través del famoso recopilatorio fotográfico «Men at work», icono de Estados Unidos en los años 70. En él se da una visión romántica de los obreros de la construcción de los grandes rascacielos, resaltando su fuerza y masculinidad al servicio del sistema. Todo ello es una cuestión simbólicamente importante, ya que la clase trabajadora, especialmente industrial, es donde se aglutinan numerosos trabajadores bajo un mismo techo y es, por definición, una amenaza al sistema capitalista por su capacidad de plantear y movilizarse por otro sistema económico que sí les sea más favorable. Es por esta razón que se produce la paradoja de que, pese a que la clase obrera es producto del capitalismo, "la posibilidad social del capitalismo está vinculada al hecho de que la fuerza de trabajo no devenga clase obrera, sujeto social alternativo, sino individuos» (Bilbao, 1995: 11).

Palermo (2015: 110) va algo más allá al argumentar que no sólo se refuerza la cultura masculina, sino que se hace de forma dicotómica frente a la feminidad, polarizando a los hombres y a las «señoritas», entendiendo, además, esta última categoría de forma peyorativa. Como señala el autor, más allá de los efectos sobre el género, estas categorizaciones se utilizan en beneficio de la rentabilidad productiva y en detrimento de la seguridad de los trabajadores. Por tanto, se da el caso de que a través de la masculinidad se evita el conflicto de clase y se unifican los intereses empresariales y obreros, por lo que la entrada de la mujer en estos entornos laborales no solo es un desafío al orden de las cosas, sino que desmonta la lógica orientada a incrementar la productividad de los trabajadores.

\subsection{La masculinidad como competencia laboral}

La gestión por competencias, al sustentarse en ocasiones en cuestiones poco objetivables y cambiantes como son la actitud de las personas trabajadoras, reduce la objetividad que se puede aportar al proceso de gestión del personal, y entra en aspectos personales, de forma que "la organización demanda al individuo no solamente sus conocimientos técnicos en un determinado campo, sino también sus comportamientos, actitudes y motivaciones» (Tovar y Revilla, 2010: 110). Teniendo en cuenta que la práctica del género implica también habilidades, es evaluado también en el proceso de selección.

De esta manera, aunque los sectores tradicionalmente masculinos empiezan a integrar cada vez a más mujeres, la masculinidad sigue englobando un conjunto de habilidades consideradas necesarias para realizar bien dichos trabajos. Podemos observar esta vinculación desde el punto de vista empírico en el ámbito petrolero en los trabajos de Palermo $(2015,2017)$ y en el de la construc- 
ción en las investigaciones de Gallioz (2009), donde se afirma que la incorporación de mujeres es compleja porque se mantiene la consideración de que no son capaces de realizar un trabajo tan duro como ése. Ello crea una brecha entre los protocolos «oficiales» y su puesta en práctica, entre la estructura material y la estructura simbólica de la empresa, que hace que no sea necesario prohibir la entrada a las mujeres, sino que en la misma búsqueda del personal mejor preparado se privilegiará la entrada de hombres.

En Stobbe (2005), vemos cómo esas concepciones de que las mujeres son menos adecuadas para el puesto de trabajo penetran en los mismos procesos de contratación, evitando que se contraten mujeres. En este sentido, coincidimos con Collins (2013) en el hecho de que la masculinidad puede ser considerada desde el punto de vista de una competencia laboral, ya que los atributos propios de la masculinidad se tendrán en cuenta en la selección de personal, así como en la capacidad de ascenso del trabajador, por lo que determinará profundamente la trayectoria laboral de la persona. En el caso de los trabajos feminizados ocurre lo mismo, como en el ejemplo de las «mantecaeras» de Estepa, un trabajo industrial que requiere de habilidades para la manipulación de productos pequeños con delicadeza, habilidades que se entienden como innatas en las mujeres y hacen que la plantilla sea totalmente femenina (Téllez, 2002).

Al tomar la perspectiva de considerar el género como una competencia laboral nos referimos muy específicamente a que en el mercado laboral se favorece a quienes se ajusten a la construcción de la masculinidad o feminidad imperante en un determinado entorno laboral, lo cual hace que no se discrimine de la misma manera en todos los hombres o mujeres, sino que se hace en función de su ajuste a los cánones de género. En el caso de la masculinidad, como bien detalla un informante en la investigación de Stobbe (2005), el machismo supone una relación de dominación, no solo hacia las mujeres, sino también hacia otros hombres. Collins (2015: 416) también hace esta observación, al determinar que «el privilegio masculino se aplica solamente a algunos hombres». Este hecho se evidencia en su estudio, en el que analiza las formas de discriminación que sufren los hombres homosexuales, que, como recoge el autor, tradicionalmente se han considerado menos masculinos. Además, las actitudes y habilidades que engloban masculinidad y feminidad y que se valoren en una empresa serán distintos en cada contexto laboral en concreto, ya que en cada una hay un "repertorio de prácticas» (Slutskaya, 2016: 167) que determina lo que es "performar» una determinada posición de género en un lugar determinado.

\subsection{Riesgo y violencia en el entorno industrial}

El riesgo es un elemento muy presente en el ámbito del trabajo industrial, donde un trabajador debe saber asumir los riesgos como un hombre. De hecho, en Stergiou-Kita et al. (2015: 216), se indica que "hacer trabajo peligroso 
es equivalente a hacer género», ya que las diferentes percepciones y formas de afrontar el riesgo son elementos centrales en las construcciones de género tradicionales. Matošević (2010: 33), por su parte, considera que el trabajo peligroso es el corazón de la masculinidad en el ámbito laboral, y Collins (2014), al establecer una distinción entre los lugares de trabajo con una mayoría de hombres y los lugares de trabajo masculinizados, considera que uno de los elementos clave para diferenciarlos es el nivel de riesgo en el lugar de trabajo. Analizando los datos, vemos sus efectos prácticos. En el caso de España, según datos del Ministerio de Empleo y Seguridad Social, vemos cómo el 86,7\% de los accidentes ocurridos en el lugar de trabajo entre enero y noviembre de 2017 les ocurrieron a hombres, y solo el 13,3\% a mujeres.

Palermo $(2015,2017)$ observa cómo el aguante del dolor es un símbolo de masculinidad, que minimiza los accidentes cuando ocurren y hace que los trabajadores muestren sus cicatrices como medallas de virilidad. Parar el trabajo por un accidente o mostrar dudas ante una situación son actitudes consideradas femeninas, y son evitadas por los trabajadores, que incluso elevan a la categoría de héroes a los trabajadores muertos en el ejercicio de su trabajo petrolero, que dota al resto de un halo de valentía al ejercer su trabajo. Se logra así aproximarse más a ese concepto abstracto de trabajador totalmente al servicio de la empresa, incluso por encima de las necesidades de su cuerpo. En la misma línea, Ness (2012: 666-667) señala cómo «tener el trabajo hecho» es un símbolo de «dureza y masculinidad» para los trabajadores, que no deja de coincidir con los objetivos de la empresa. Obreros que lo último que querrían sería una mejora de las condiciones laborales y de seguridad, ya que la idea de que "demasiado confort es blando aún persiste en la cultura del lugar».

Ello apunta a reforzar, especialmente a través de este elemento del riesgo, una dominación de clase, según la cual la empresa obtiene beneficios en el uso de la cultura de la masculinidad obrera (Palermo, 2015). Stergiou-Kita et al. (2015) hablan de que los cuerpos de los hombres trabajadores se convierten en un "capital corporal» desde el punto de vista de sus superiores, que disponen de sus cuerpos en pos de los beneficios empresariales. Así, disciplina fabril y virilidad se conjugan para mejorar la eficiencia productiva a través de reducir el tiempo perdido y las bajas laborales (aunque no disminuyan los accidentes). El espíritu competitivo y la cultura del trabajo incluidos en la masculinidad obrera están muy basados en el movimiento stakhanovista (Matošević, 2010), que se basa en aumentar la producción de la empresa a costa del propio sacrificio, de forma que se superponen los intereses empresariales con los del trabajador, eliminando el conflicto de clase a través de la masculinidad.

Masculinidad y feminidad no son elementos únicamente vinculados a la división de sexos, sino que también «son posiciones relativas íntimamente vinculadas a los contextos situados» (Palermo, 2017: 120). La violencia es un recurso 
utilizado para mantener posiciones de dominación masculina, por lo que «la violencia de género es un ejercicio de poder» (Varela, 2017: 105). Y el acoso o la violencia sexual son mecanismos violentos de dominación que siguen la misma lógica, por lo que la motivación no parte del deseo sexual, sino de la reafirmación del poder. Por ello, como también defienden McLaughlin et al. (2012: 626), «los hombres pueden ser vulnerables al acoso si ellos son percibidos como femeninos (...) y las mujeres pueden ser el objetivo si ellas retan su posición subordinada en el sistema de género». En Palermo (2017: 118), encontramos distintos ejemplos sobre la violencia en el entorno laboral. Las «jodas» o «bromas pesadas» aparecen como habituales en su trabajo de campo, y algunas de ellas «se sitúan en un límite muy delgado entre la picardía colectiva y la violencia, y muchas veces se encuentran cargadas de connotaciones sexuales». También relata la existencia de casos de violación hacia otros hombres, como prácticas disciplinadoras sobre la feminidad que algunos hombres (normalmente los que llevan menos tiempo en el trabajo) parecen tener. Estas prácticas parecen ser combinadas con la homofobia existente en este ámbito laboral ya que se ejerce desde una posición activa de dominación. Para que el acoso sexual pueda tener lugar, según Pérez del Río (2007: 188) deben darse tres condiciones: «el secreto de las actuaciones, la connivencia de los compañeros (testigos mudos) y el sentimiento de vergüenza y humillación e incluso de culpabilidad de la víctima», condiciones reforzadas por la camaradería propia de la masculinidad obrera.

\section{Las dificultades de incorporar a las mujeres en el ámbito del trabajo industrial}

\subsection{La exclusión de la feminidad}

Mediante el proceso de racionalización de la producción industrial, la emocionalidad es alejada simbólicamente del lugar de trabajo, lo cual incluye la represión de toda dimensión corporal poco controlable. En las prácticas fordistas «la distancia emocional es condición sine qua non para su funcionamiento eficiente» (Guash, 2006: 59), lo que supone una deshumanización de las organizaciones productivas. Para un mayor control de las emociones y la reproducción, las fábricas siguieron los modelos de grandes organizaciones anteriores, como los ejércitos o los monasterios, excluyendo a las mujeres de dichos espacios y estableciendo una fuerte represión de la sexualidad, lo que facilitaba el control de sus miembros (Acker, 2000). El mismo Gramsci consideraba que «el nuevo tipo de hombre que la racionalización de la producción exige no puede crearse hasta que se haya regulado apropiadamente el instinto sexual» (Elley, 2003: 201).

Esta represión supone la expulsión de quienes son consideradas portadoras de la emocionalidad e incitadoras a la sexualidad, las mujeres. Ello ya es ob- 
servado por Federici (2010: 263) en los siglos XVI y XVII, cuando «una mujer sexualmente activa constituía un peligro público, una amenaza al orden social ya que subvertía el sentido de responsabilidad de los hombres y su capacidad de trabajo y autocontrol». Todo ello se da en un momento en el que la ubicación de la producción en las fábricas materializó fuertemente la división hogar-fábrica, lo cual, unido a la prohibición del empleo infantil en el siglo XIX y las largas jornadas laborales, problematizó seriamente a las familias el mantenimiento de las tareas de reproducción. Por ello, las clases media y alta, junto con los hombres trabajadores fueron empujando a que las mujeres fueran eliminadas de las fábricas (Hartmann, 1994). Unas fábricas que en un primer momento se habían nutrido de trabajadoras mujeres y mano de obra infantil, consideradas mucho más disciplinables que los hombres (Marglin, 1974).

Esta exclusión ha sido rápidamente extinguida en los momentos de necesidad, y tenemos buen ejemplo de ello son los periodos de Segunda Guerra Mundial, en los que los hombres abandonaron las fábricas para luchar en los frentes y fueron las mujeres las que llenaron esos puestos de trabajo. La provisionalidad de la situación quedó clara con el regreso de los hombres, que recuperaron su papel de trabajadores fabriles, y las mujeres su papel como ama de casas (Varela, 2017: 178). Este proceso es indesligable de la organización de la familia moderna mediante el modelo breadwinner. Este modelo aún es observable en la actualidad, en que, aunque las mujeres cada vez tienen una mayor presencia en el ámbito laboral remunerado (Morini, 2014), la dedicación a las tareas de reproducción es, aún hoy, «una pieza clave para entender la débil o precaria presencia laboral, cuando no la ausencia, de las mujeres europeas de entre 25 y 54 años» (Torns, 2005: 19).

Cuidados y familia también son separados del entorno industrial. Vemos un ejemplo en las observaciones de Palermo (2015: 110) sobre los trabajadores de los pozos petrolíferos de Comodoro Rivadavia, que «son espacios distantes de instituciones como el matrimonio y la paternidad, que acercan a los hombres, de alguna forma, a la feminidad que se encuentra fuera del espacio de la producción». Esta exclusión no es solo física en el lugar de trabajo, sino que también se produce en la misma vida de los trabajadores, a los que se les supone libres de cargas familiares (Ness, 2012: 668). Familia, crianza, cuidados o tareas domésticas no parecen tener nada que ver con el trabajador industrial, movido por otras lógicas. Los mismos requerimientos de eficiencia productiva de la organización fordista parecían imponer que, especialmente una vez casadas, las mujeres se dedicaran en exclusiva al trabajo reproductivo, lo que suponía su desaparición de los lugares de trabajo (Federici, 2010). De este modo, ser un trabajador masculino en el lugar de trabajo parece comportar implícitamente la necesidad de ayuda externa, normalmente de una mujer, que resuelva las tareas de reproducción y cuidado del mismo trabajador y del resto de la familia. Palermo (2017) relata cómo las mujeres hacen posible mantener las exigencias de la disciplina fa- 
bril en el entorno doméstico, asegurando que los trabajadores descansen lo suficiente o tengan lo necesario preparado para poder volver a tiempo al trabajo.

La organización de la clase obrera en el capitalismo incipiente también se construyó sin las mujeres, lo que se observa en las dificultades que históricamente han tenido las organizaciones obreras para afiliar y representar a las trabajadoras femeninas. La complicada relación histórica entre el feminismo y el socialismo es un buen ejemplo de la dificultad de articular la condición obrera con las aspiraciones de la igualdad de género en el ámbito laboral. Elley (2003), Varo (2014) y Wikander (2016) nos muestran numerosos ejemplos de las contradicciones que históricamente han tenido partidos y sindicatos para representar a las mujeres, así como las ambivalentes posiciones que ha tomado el feminismo frente a las aspiraciones vindicativas obreras. Las mujeres han tenido muchas dificultades para ser consideradas en su dimensión de trabajadoras, como pone de manifiesto Varo (2014: 96) en su estudio sobre la participación de las mujeres en Comisiones Obreras:

No quedaba claro que las protestas de enfermeras o maestras fuesen protestas obreras. Por otra parte, las trabajadoras industriales tendían a ser tratadas como víctimas anónimas de los empresarios o de las fuerzas de seguridad (a quienes los hombres debían defender) o eran masculinizadas como 'trabajadores' para poder asimilarlas a esta clase obrera heroica.

Así, «se construyó un ideal de virilidad y dignidad de la clase trabajadora como respuesta a las privaciones de clase y a las estrategias paternalistas de gestión, mientras mediante las mismas acciones se definía contra las mujeres trabajadoras» (Connell, 1995: 10).

Para Federici (2010), la exclusión de las mujeres responde a diversos factores: la tradición de salarios más bajos para las mujeres que las convertía en una competencia desleal, el menor nivel educativo femenino y la mayor organización de los hombres en ese momento de capitalismo incipiente. Ello es recogido por Marglin (1974) en el sector de la fabricación textil, donde, según el autor, ante la competencia laboral femenina los hombres pactaron colectivamente que evitarían que las mujeres aprendieran el oficio. Hartmann (1994: 274) recoge que incluso "la hostilidad hacia la competencia de las mujeres jóvenes, casi seguramente menos calificadas y menos bien pagadas, era bastante corriente».

En todo caso, es importante remarcar que no fue tan fácil que las mujeres dejaran el trabajo remunerado, ya que, como ya señalaba Drucker (1911: 10) a principios de siglo «nunca se les ha ocurrido la idea de que el sueldo de uno solo pudiera ser el equivalente del consumo de ocho o diez personas o más». Y es que la idea de salario familiar, un salario que sirve para mantener a toda la familia, para la clase trabajadora era una utopía, lo cual hace que las mujeres nunca hayan dejado de estar en el ámbito productivo, aunque invisibilizadas. Así, la ex- 
clusión de la feminidad en el mercado laboral no se ha podido aplicar nunca del todo en la práctica, sino que ha sido «disimulado», según palabras de Wikander (2016: 11), por los miembros de la pareja. En este sentido, "la división del trabajo según sexos vino a ser una salida al dilema entre el salario necesario de la mujer y el ideal del hombre como sostén». Así, a través de los trabajos femeninos y masculinos, las mujeres podían entrar al mercado laboral a través de trabajos que no compitieran con los de sus maridos. Por tanto, la presencia femenina en el ámbito laboral se da pues en un mercado segmentado, con sectores privilegiados reservados a los hombres, y sectores precarizados con mayor presencia de las mujeres.

\subsection{La masculinización como resolución de los conflictos entre género e identidad laboral en las mujeres}

Las investigaciones actuales muestran cómo esa historia del trabajo industrial en masculino aún mantiene barreras a la entrada de las mujeres. La identificación constante de sus tareas como trabajos menos duros e importantes a lo que hacíamos referencia en el apartado anterior hace que las trabajadoras se sientan como «outsiders» en esas empresas, con la sensación de no estar del todo integradas. Miller (2004) recoge entre las mujeres de la industria un sentimiento de ser «outsiders» de ese espacio. Este conflicto, surgido de la interiorización de estar realizando un trabajo que no es adecuado para la propia "performance» de género, supone muchas veces una colisión de la identidad de género y la identidad laboral. La misma autora detalla cómo las mujeres ingenieras usan estrategias para que su feminidad pase inadvertida entre el resto de trabajadores masculinos. Y es que en ese entorno laboral se da una clara distinción entre los roles femeninos y los roles «normales» en el ejercicio de la ingeniería, naturalizando como neutrales o normales los masculinos y los femeninos como no normales, diferentes. En sus testimonios, ello es interiorizado por las informantes, que expresan un claro conflicto entre su identidad femenina y su identidad laboral como ingenieras. Estas mujeres apuestan por ser consideradas ingenieros normales en el entorno laboral, y, por tanto, masculinizan sus actitudes y comportamientos.

Estos resultados coinciden con los de García-Retamero y López-Zafra (2006) en las mujeres que ejercen el liderazgo empresarial, un ámbito también tradicionalmente masculino. En su estudio observan que los hombres generalmente cuentan con más influencia en los grupos y que ello provoca una adopción de las características tradicionalmente masculinas por parte de las mujeres para conseguir una mayor autoridad y ser reconocidas como lideresas. Por ello podemos afirmar que esta estrategia femenina es común en entornos laborales tradicionalmente masculinos, además de la industria. En la investigación de Beraud (2007: 157) las mujeres ingenieras sí se identifican como tales, pero esta identidad «es continuamente cuestionada por la experiencia de la vida profesio- 
nal, que se encarga de recordarles a las mujeres que ellas son mujeres y que en nuestra sociedad ellas están todavía al cargo de la educación de los hijos». En el estudio de Holmes y Stubbe (2003), podemos observar cómo las mujeres que quieren ascender también incorporan unas formas de comunicarse más masculinas, que parecen ser necesarias para obtener un mayor respeto de sus compañeros y subordinados.

En cambio, los resultados de Miller (2004) indican una realidad muy diferente para los hombres que trabajan en entornos laborales tradicionalmente femeninos. En su investigación, los hombres también experimentan ese conflicto entre su identidad masculina y su identidad laboral, pero priorizan que se les reconozca como hombres. Así pues, sus estrategias van en el sentido contrario, priorizando reafirmar su identidad masculina o, en algunos casos, reconvertirla de manera que no entre en conflicto con la identidad laboral. Esta distinción entre las maneras de resolver la misma contradicción en hombres y mujeres puede tener que ver con salvaguardar la identidad más valiosa, la que supone un mayor estatus social y da lugar a un mayor nivel de poder, que en el caso de las mujeres es la identidad laboral y en el caso de los hombres, la masculina. En Simpson (2004) observamos esas consideraciones especiales hacia los hombres que trabajan en profesiones feminizadas. Concretamente, la autora observa que la masculinidad les aporta una mayor consideración, una mayor autoridad y su actitud se percibe como más orientada a los cuidados por ser poco habitual en hombres.

En las investigaciones empíricas las razones de la exclusión de las mujeres en entornos laborales masculinizados son variadas, pero generalmente coincidentes. Por ejemplo, Stobbe (2005) encuentra tres tipos de discursos para explicar la exclusión de las mujeres en los procesos de selección que ella analiza: (1) que las mujeres no se adecuaban bien al puesto de trabajo, (2) que las mujeres son menos válidas y (3) que son más problemáticas como trabajadoras que los hombres. En todos estos discursos subyace la contradicción entre feminidad y trabajo. En este sentido, Ness (2012) analiza la exclusión de las mujeres en el sector de la construcción y señala que sus informantes consideran que las mujeres serían una distracción para sus empleados hombres y que supondría quebrantar una camaradería masculina existente. Además, en muchos casos, hay un cierto conservadurismo que se formula en que simplemente no quieren lidiar con los conflictos que podrían aparecer al contratar a un trabajador no masculino y que asumen las actitudes sexistas que tendrían los trabajadores hombres como inevitables.

En el estudio de Denissen (2010) se observa claramente el conflicto entre feminidad y trabajo en las propias mujeres trabajadoras en entornos masculinizados, que viven una contradicción entre las distintas expectativas de cada una de las dos identidades. Las mujeres entrevistadas desarrollan diferentes estrategias mediante las cuales resolver estas contradicciones, utilizando la identidad laboral o la identidad de género en función de la situación, que favorece que «traba- 
jen como un hombre» o que se hagan «respetar como mujeres». En Aguilar-Cunill (2018), para el sector de la industria química, también encontramos estas contradicciones, que parten de las resistencias masculinas a la incorporación de mujeres en este entorno laboral, y que se traducen más tarde en situaciones de acoso por razón de género o incluso sexual en el entorno laboral. Sin embargo, en la investigación de Gallioz (2009) encontramos también la paradoja de que la incorporación de mujeres tiene una clara voluntad de romper con el modelo únicamente masculino, al que se identifica como anticuado. De esta manera, la contratación de mujeres viene acompañada de un conjunto de cambios orientados a materializar esta modernización del sector de la construcción. Si bien en este caso el género es un elemento que adquiere un valor añadido, el género, concretamente el femenino, mantiene una carga simbólica que va más allá de la normalidad de contratar a personas que puedan ser mujeres y hombres, es una declaración de intenciones y una ruptura con la naturalización de tener unas plantillas únicamente masculinas.

\section{Conclusiones}

A lo largo del artículo hemos expuesto, desde una perspectiva interseccional, cómo el género, junto con la identidad de clase obrera, han sido claves en la concepción y organización del trabajo industrial. El desarrollo de un determinado modelo de masculinidad obrera ha permitido mantener la masculinización del sector industrial que encontramos todavía en la actualidad. Hemos observado también que este modelo supone el privilegio de un determinado modelo de hombre frente a los otros mediante la exclusión de todo aquello vinculado a la feminidad, que no sólo afecta a las mujeres, sino también a determinados modelos de masculinidad. La idealización de un determinado tipo de masculinidad en la organización del trabajo industrial tiene que ver con el hecho de que es en los entornos laborales «donde las masculinidades son construidas, redefinidas y puestas a prueba» (Lupton, 2000: 34), al constituir un espacio fundamental para la construcción de la masculinidad (Connell, 1995), e incluso se lleva al nivel laboral la verificación constante de la virilidad que se da entre los hombres en las demás esferas de la vida (Kimmel, 1992).

Como reflejan ampliamente los Men's studies, las masculinidades son diversas y suponen posiciones de género en relación no solo a las mujeres, sino también entre hombres. Por ello, a lo largo del texto nos referimos a la masculinidad y la feminidad como prácticas sociales performativas, entendiendo que los privilegios y las discriminaciones laborales no están vinculadas a hombres y mujeres, sino a determinadas formas de masculinidad y feminidad, que no siempre son corporeizadas por hombres y mujeres respectivamente, sino que corresponden a distintas formas de "hacer género" (West y Zimmerman, 1987). Formas que 
muestran como la dominación no es sólo masculina, sino de un sujeto masculino heterosexual que mantiene sus privilegios mediante distintas jerarquizaciones (de etnia, clase, género e identidad sexual). Por esto, desde la perspectiva de Ziga (2009: 30), el feminismo sin esta perspectiva es blanco, heterosexual y burgués, igual que sin la noción critica del sexo y del género, es esencialista y tránsfobo, pues «comulga de alguna manera con toda la violencia a través de la que se nos sigue tratando de moldear como hombres o mujeres».

Entendiendo que las discriminaciones de género en el ámbito laboral son clave para la construcción y reproducción de las desigualdades en el resto de la sociedad, al ser una sociedad en la que el salario es la principal fuente de recursos y de subsistencia, es éste un ámbito clave para el desarrollo de mejoras en pos de la igualdad. En la dirección de estas mejoras, este artículo señala dos elementos a tener en cuenta en próximas investigaciones. En primer lugar, la importancia de avanzar en el análisis de la igualdad de género en el trabajo a través de la integración de una perspectiva interseccional para analizar no sólo las identidades discriminadas, sino también los modelos de masculinidad que son privilegiados. Una perspectiva que también seria clave integrar en las medidas de intervención que se planteen. En segundo lugar, las posibilidades teóricas y de intervención que existen en el entorno laboral industrial, que se mantiene como un entorno laboral muy vinculado a la masculinidad, en el que las construcciones de género se mantienen fuertemente unidas a los fundamentos de la organización misma del trabajo. Por ello pensamos que profundizar en estos espacios puede darnos muchos elementos de análisis muy valiosos que podrían servir también para otros entornos laborales que, aunque sean mixtos, también privilegian a los trabajadores masculinos.

\section{Referencias bibliográficas}

ACKer, Joan (2000): "Jerarquías, trabajos y cuerpos: una teoría sobre las organizaciones dotadas de género», en Navarro, M. y Stimpson, C. (comp.), Cambios sociales, económicos y culturales, El Salvador, Fondo de Cultura Argentina, 111-139.

ACKer, Joan (2006): «Inequality regimes: Gender, Class and race in organizations», Gender and Society, 20(4), 441-464.

Aguilar-Cunill, Carla (2018): «Disimular la feminidad, vestirse de masculinidad. Mujeres operadoras de la industria química de Tarragona», Revista Internacional de organizaciones, 20, 123-146.

Arbaiza, Mercedes (2014): «Obreras, amas de casa y mujeres liberadas. Trabajo, género e identidad obrera en España», en Nash, M. (coord.), Feminidades y masculinidades. Arquetipos y prácticas de género, Madrid, Alianza Editorial, 129-158.

Archettr, Eduardo (1999): Masculinidades. Fútbol, tango y polo en la Argentina, Buenos Aires, Antropofagia.

Badinter, Elisabeth (1993): XY. La identidad masculina, Madrid, Alianza. 
Banco Mundial (BM) (2018): Employment in industry, men. Recuperado de: https:// data.worldbank.org/indicator/SL.IND.EMPL.MA.ZS?year_high_desc=false.

BEARD, Mary (2018): Mujeres y poder. Un manifiesto, Barcelona, Crítica.

Beraud, André (2007): La llegada de las mujeres a las actividades tradicionalmente masculinas. ¿transformación de las identidades profesionales?, en Guadarrama, R. y Torres, J. L. (coords.): Los significados del trabajo femenino en el mundo global, Barcelona, Anthropos.

BilbaO, Andrés (1995): Obreros y ciudadanos la desestructuración de la clase obrera. Madrid, Trotta, Fundación 1 de Mayo.

Blanco-Cano, Rosana (2017): "¿Nuevas masculinidades en la televisión mexicana contemporánea?: El sexo débil (2011)», Estudios de Genero de El Colegio de México, 3(6), 101-126.

Brunet, Ignasi y SAntamaria, Alberto (2018): «Gender system and organizations». Revista internacional de organizaciones, 20, 11-39.

Butler, Judith (2002): Cuerpos que importan. Sobre los limites materiales y discursivos de sexo, Buenos Aires, Paidós.

ButLer, Judith (2006): Deshacer el género, Barcelona, Paidós.

ButLER, Judith (2007): El género en disputa, El feminismo y la subversión de la identidad, Barcelona, Paidós.

Claveria, Silvia (2018): El feminismo lo cambia todo. Un relato sobre la lucha contra el patriarcado, Barcelona, Paidós.

Collins, Joshua C. (2013): «Stress and safety for gay men at work within masculinized industries», Journal of Gay \& Lesbian Social Services, 25, 245-268.

Collins, Joshua C. (2015): «Characteristics of «Masculinized» Industries: Gay Men as a Provocative Exception to Male Privilege and Gendered Rules», Human Resource Development Review, 14(4), 415-441.

Comas, Dolors (1995): Trabajo, género, cultura: la construcción de desigualdades entre hombres y mujeres, Barcelona, Icària.

Connell, Raewyn W. (1995): Masculinites, Berkeley, University of California Press.

Connell, Raewyn W. (1995): La organización social de la masculinidad, en Olavarría, J. y Valdés, T. (eds.) Masculinidadles: poder y crisis, Santiago de Chile, Isis Internacioal/ FLACSO, 31-48.

CoRIAT, Benjamin (1982): El taller y el cronómetro: ensayo sobre taylorismo, fordismo y la producción en masa, Madrid, Siglo XXI.

CoRIat, Benjamin (1992): El taller y el robot. Ensayos sobre el fordismo y la producción en masa en la era electrónica, Madrid, Siglo XXI.

Crenshaw, Kimberlé Williams (1991): «Mapping the Margins: Intersectionality, Identity Politics, and Violence against Women of Color», Stanford Law Review, 43(6), 12411299.

Denissen, Amy M. (2010): «The right tools of the job: constructing gender meanings and identities in the male-dominated building trades», Human Relations, 63(7), 1051-1069.

DruCKer, Wilhelmina (1911) : Autour du travail de la femme, Amsterdam.

Elley, Geoff (2003): Un mudo que ganar: historia de la izquierda en Europa, Barcelona, Crítica.

Embrick, David G., Walther, Carol S. y Wickens, Corrine M. (2007): «Working class masculinity: keeping gay men and lesbians out of the workplace», Sex Roles, 56(11-12), 757-766. 
Esteban, Mari Luz (2011): Critica del pensamiento amoroso, Barcelona, Edicions Bellaterra.

Federici, Silvia (2010): Calibán y la bruja. Mujeres, cuerpo y acumulación originaria, Madrid, Traficantes de Sueños.

Federici, Silvia (2013): Revolución en Punto Cero, Madrid, Traficantes de Sueños.

Foucault, Michel (1999): Estrategias de poder. Obras esenciales, Barcelona, Paidós.

Foucault, Michel (2007): Nacimiento de la biopolitica. Curso en el Collège de France (1978-1979), Buenos Aires, FCE.

Fuller, Norma (1997): Fronteras y retos: Varones de clase media del Perú, en Valdés, T. y Olavarría, J. (ed.), Masculinidadles. Poder y crisis, Santiago de Chile, Isis Internacional.

Gallioz, Stéphanie (2009) : "La féminisation dans les entreprises du bâtiment : une normalisation sociale des comportements ouvriers masculins? ", Cahiers du Genre, 47(2), 55-75.

García-Retamero, Rocío y López-Zafra, Esther (2006): «Prejudice against Women in Male-congenial environments: perceptions of gender role congruity in leadership", Sex Roles, 55, 51-61.

Gil Calvo, Enrique (2006): Máscaras masculinas. Héroes, patriarcas y monstruos, Barcelona, Anagrama.

Gilmore, David (1994): Hacerse hombre: concepciones culturales de la masculinidad, Barcelona, Paidós.

Guash, Oscar (2006): Héroes, cientificos, heterosexuales y gays. Los varones en perspectiva de género, Barcelona, Edicions Bellaterra.

Guash, Oscar (2008): «Los varones en perspectiva de género. Teorías y experiencias de discriminación", Asparkia, 19, 29-38.

Hartmann, Heidi (1994): Capitalismo, patriarcado y segregación de los empleos por sexos, en Borderías, C., Carrasco, C. y Alemany, C., Las mujeres y el trabajo. Rupturas conceptuales, Barcelona, Icaria/FUHEM, 255-294.

Holmes, Janeth y Stubbe, Maria (2003): Feminine Workplaces: Stereotype and Reality , en Holmes, Janeth y Meyerhoff, Miriam (eds.), The Handbook of Language and Gender, Maiden, Blackwell Publishing Ltd.

Huerta, Fernando (1999): El juego del hombre. Deporte y masculinidad entre obreros, México DF, Valdés Editores.

Kanter, Rosabeth Moss (1977): Men and women of the corporation, New York, Basic Books.

Kelan, Elisabeth K. (2010): «Gender logic and (Un) doing gender at work», Gender, Work and Organization, 17(2), 174-194.

Kimmel, Michael S. (1997): Homofobia, temor, vergüenza y silencio en la identidad masculina. en Valdés, Teresa y Olavarría, José (ed.), Masculinidadles: poder y crisis, Santiago de Chile, Isis Internacioal/FLACSO, 49-62.

KImMEL, Michael S. (1992): La producción teórica sobre la masculinidad: nuevos aportes, Santiago de Chile, Editorial de las Mujeres.

LAClaU, Ernesto (2000): Nuevas reflexiones sobre la revolución de nuestro tiempo, Buenos Aires, FCE.

Lamas, Marta (1994): «Cuerpo: diferencia sexual y género», Debate feminista, 10, 3-31.

Lamas, Marta (Comp.) (2008): El género: la construcción cultural de la diferencia sexual, México DF, PUEG.

List Reyes, Mauricio (2005): Hombres: cuerpo, género y sexualidad, Cuicuilco, 12, 173202. 
Longo, Maria Eugenia (2009): «Género y trayectorias laborales», Trayectorias, 11(28), 118-141.

Lupton, Ben (2000): «Mantaining Masculinity: Men who do «Women’s Work»». British Journal of Management, (11)28, pp.S33-S48.

Marglin, Stephen A. (1974): ¿Qué hacen los patrones? Orígenes y funciones de la jerarquía en la producción capitalista, en Marglin, S., Perdiendo el contacto. Hacia la descolonización de la economía, Lima, CAM, 19-69.

MatošEvić, Andrea (2010): «Industry Forging Masculinity. «Tough men» and hard labour». Narodna Umjetnost, 47(1), 29-47.

McLaughlin, Heather, Uggen, Christopher y Blackstone, Amy (2012): «Sexual harassment, workplace authority, and the paradox of power», American Sociological Review, 77(4), 625-647.

Miller, Gloria E. (2004): «Frontier masculinity in the oil industry: the experience of women engineers", Gender, Work and Organization, 11(1), 47-73.

Ministerio de empleo y Seguridad social de España, Estadística de accidentes de trabajo, avance enero-noviembre 2017. Recuperado de: http:/www.empleo.gob.es/estadisticas/eat/welcome.htm

Moral, David y Brunet, Ignasi (2018): «La imagen del sindicato en el siglo xxi a la luz de su contestación por los Nuevos Movimientos Sindicales», Sociología Del Trabajo, 93, 307-326.

Morini, Cristina (2014): Por amor o a la fuerza. Feminización del trabajo y biopolitica del cuerpo, Madrid, Traficantes de Sueños.

Mosse, George L. (2001): La imagen del hombre. La creación de la moderna masculinidad, Madrid, Talasa.

Nash, Mary (ed.) (2014): Feminidades y masculinidades. Arquetipos y prácticas de género, Madrid, Alianza.

Ness, Kate (2012): «Constructing Masculinity in the Building Trades: «Most Jobs in the construction Industry can be done by Women"», Gender, Work and Organization, 19(6), 654-676.

Organización Internacional del Trabajo (OIT) (2016): Las mujeres en el trabajo. Tendencias de 2016, Ginebra, OIT.

PALermo, Hernán (2015): ««Machos que se la bancan»: masculinidad y disciplina fabril en la industria petrolera argentina», Desacatos, 47, 100-115.

Palermo, Hernán (2017): La producción de la masculinidad en el trabajo petrolero, Buenos Aires, Editorial Biblos.

Palermo, Hernán (2018): «Masculinidades en la industria del software en Argentina», Revista Internacional de Organizaciones, 20, 103-122.

Pantelides, Alejandra y LóPez, Elsa (Comps.) (2005): Varones Latinoamericanos, Buenos aires, Paidós.

Pazos, María (2018): Contra el patriarcado. Economía feminista para una sociedad justa y sostenible, Pamplona, Katakrak Liburuak.

PÉRez del Río, Maria Teresa (2007): «La violencia de género en el trabajo: el acoso sexual y el acoso moral por razón de género", Temas laborales, 91, 175-203.

Pérez-Orozco, Amaia (2004): «Estrategias feministas de deconstrucción del objeto de estudio de la economía», Foro Interno: Anuario de Teoría Politica, 4, 87-117.

Pérez Orozco, Amaia (2014): Subversión feminista de la economía, Madrid, Traficantes de Sueños.

Perry, Grayson (2018): La caida del hombre, Barcelona, Malpaso Ediciones. 
Peterson, Helen (2007): "Gendered work ideals in Swedish IT Firms: Valued and not valued workers», Gender, work and organization, 14(4), 333-348.

Pineda-Duque, Javier (2000): Masculinidades y desarrollo. El caso de los compañeros de las mujeres cabeza de hogar, en Robledo, A. y Puyana, Y., Ética: masculinidades y feminidades. Bogotá, Universidad Nacional de Colombia.

Preciado, Paul B. (2016): Manifiesto contrasexual, Barcelona, Anagrama.

Romero, Carmen (2003): «De diferencias, jerarquizaciones excluyentes, y materialidades de lo cultural. Una aproximación a la precariedad desde el feminismo y la teoría», Cuadernos de Relaciones Laborales, 1, 33-60.

Salinas, Hector Miguel (2016): Masculinidades e identidades Gay: Tres estudios sobre violencia, mercado y sociabilidad gay en la Ciudad de México, México, Voces en Tinta.

Sanchez-Palencia, Carolina e Hidalgo, Juan Carlos (eds.) (2001): Masculino plural. Construcciones de la masculinidad, Lleida, Universidad de Lleida.

Segarra, Marta y Carabí, Àngels (eds.) (2000): Nuevas masculinidades, Barcelona, Icaria.

Seidler, Victor (2006): Transforming Masculinities: Men, Cultures, Bodies, Power, Sex and Love, London, Routledge.

Serra, Clara (2018): Leonas y zorras. Estrategias politicas feministas, Madrid, Catarata.

Simpson, Ruth (2004): «Masculinity at work: the experiences of men in female dominated occupations», Work, employment and society, 18(2), 349-368.

Slutskaya, Natasha, Simpson, Ruth, Hughes, Jason, Simpson Alex, y Uygur, Selçuk (2016): "Masculinity and class in the context of dirty work», Gender, Work and Organization, 23(2), 165-182.

Soley-Beltran, Paticia (2009): Transexualidad y la matriz heterosexual. Un estudio critico de Judith Butler, Barcelona, Edicions bellaterra.

Stergiou-Kita, Mary, Mansfield, Elisabeth, Bezo, Randy, Colantonio, Angela, Garritano, Enzo, Lafrance, Marc, Lewko, John, Mantis, Steve, Moody, Joel, Power, Nicole, Theberge, Nancy, Westwood, Eleanor y Travers, Krista (2015): "Danger Zone: Men, masculinity and occupational health and safety in high risk occupations», Safety Science, 80, 213-220.

Sтовве, Lineke (2005): «Doing Machismo: legitimating Speech Acts as a Selection Discourse», Gender, Work and Organization, 12(2), 105-123.

TÉllez, Anastasia (2001): «Trabajo y representaciones ideológicas de género. Propuesta para un posicionamiento analítico desde la antropología cultural», Gazeta de Antropología, 17, 1-14.

TÉlLEZ, Anastasia (2002): «Trabajo, identidad y género: la puesta en juego de las representaciones ideológicas», Trabajo, 20(1), 191-214.

Torns, Teresa (2005): «De la imposible conciliación a los permanentes malos arreglos». Cuadernos de Relaciones Laborales, 23(1), 015-033.

Tovar, Francisco José y Revilla, Juan Carlos (2010): «La supuesta neutralidad de la evaluación por competencias", Revista internacional de Organizaciones (RIO), 5, 109-126.

Tzul, Gladys (2015): El Patriarcado del Salario: «Lo que llaman amor, nosotras lo llamamos trabajo no pagado, Prensa Comunitaria K. 169. Recuperado de: https:// comunitariapress.wordpress.com/2015/04/21/el-patriarcado-del-salario-lo-quellaman-amor-nosotras-lo-llamamos-trabajo-no-pagado/

VARELA, Nuria (2017): Íbamos a ser reinas: mentiras y complicidades que sustentan la violencia contra las mujeres, Madrid, Ediciones B.

Valdes, Teresa y Olavarría, José (1998): Masculinidades y equidad de género en América Latina, Santiago de Chile, FLACSO. 
Valdes, Teresa y Olavarría, Jose (ed.) (1997): Masculinidad/es. Poder y crisis, Santiago de Chile, Isis Internacional.

Varo Moral, Nadia (2014): Las militantes ante el espejo: clase y género en las CCOO del área de Barcelona, 1964-1978, Barcelona, Fundación Cirpiano García de CCOO de Catalunya.

WarD, Michael R.M. (2015): «The Chameleonisation of Masculinity: Jimmy's Multiple Performances of a Working-Class Self», Masculinities and Social Change,4(3), 215240.

West, Candance y Zimmerman, Don H. (1987): «Doing Gender». Gender and Society, $1(2), 125-151$.

Wikander, Ulla (2016): De criada a empleada. Poder, sexo y división del trabajo (17891950), Madrid, Siglo XXI.

Zerilli, Linda M. G. (2008), El feminismo y el abismo de la libertad, Madrid, FCE.

Ziga, Itziar (2009), Devenir perra, Barcelona, Editorial Limusina. 